\title{
LUT
}

University

\section{A method of system model improvement using TRIZ function}

\author{
Efimov-Soini Nikolai, Elfvengren Kalle
}

This is a Final draft version of a publication

published by Palgrave Macmillan, Cham

in Advances in Systematic Creativity

DOI: $10.1007 / 978-3-319-78075-7 \_8$

Copyright of the original publication: (c) The Author(s) 2019

Please cite the publication as follows:

Efimov-Soini N., Elfvengren K. (2019) A Method of System Model Improvement Using TRIZ Function Analysis and Trimming. In: Chechurin L., Collan M. (eds) Advances in Systematic Creativity. Palgrave Macmillan, Cham

This is a parallel published version of an original publication. This version can differ from the original published article. 


\title{
A method of system model improvement using TRIZ function analysis and trimming
}

\author{
Nikolai Efimov-Soini and Kalle Elfvengren \\ Lappeenranta University of Technology, School of Business and Management
}

\begin{abstract}
This chapter presents a method of system model improvement by means of system complexity reduction, based on TRIZ function analysis and trimming. The suggested method can be used for new development as well as improvement of an existing system. The presented approach is illustrated by an industrial case study.
\end{abstract}

Keywords: TRIZ, function analysis, trimming.

\section{Introduction}

The chapter concerns a function-based method for design improvement and new design development. According to Ullman, $75 \%$ of product cost is defined at the conceptual stage, and the cost of product improvement grows exponentially in the manufacturing stage, but the use of systematic methods makes it possible to minimize the funds lost (Ullman 2010). This means that these stages are extremely important in the product life-cycle, and the use of systematic methods at these stages is thus very useful for design development.

Several systematic approaches exist, e.g. Axiomatic Design (Suh 1990), USIT (Sickafus 1997), and TRIZ (Altshuller 1984). In this chapter, the TRIZ methodology is used for design development, because it is easy to use and understand. TRIZ utilizes formal approaches and inventive tools, and it is widely used in science and industry (Luo et al. 2012; Di Gironimo et al. 2013; Chechurin 2016).

TRIZ, in Russian Theoria Resheniya Izobretatelskih Zadech, or Theory of Inventive Problem Solving, is an inventive method proposed by the Soviet inventor Genrich Altshuller in 1956 (Altshuller and Shapiro 1956). He studied about 40000 patents and drew out the formal processes for some new ideas of the generation and the technical evolution trends. The method has 40 inventive principles, contradictions, ideality, and patterns of evolution.

This chapter concerns modern TRIZ tools, such as function analysis and trimming (Gadd 2011). The latter is a formal method for system development and improvement, based in the reduction of system complexity. Different types of this tool are used for patent-around design (Li et al. 2015), system improvement (Sheu and Hou 2013), and to form new design patterns (Efimov-Soini and Uzhegov 2017). In this chapter, the advanced method of trimming is used for system improvement and development in a formal manner. Function analysis is used as the input to the trimming process.

Previous trimming methods used formal rules for step-by-step improvement of the system (Ikovenko et al. 2005; Sheu and Hou 2013; Li et al. 2015). The functions were independent in these approaches, and the authors did not use a special formal ranking index to define the importance of the function in the system. In contrast to the previous methods, the new approach takes the relation between the functions and elements into account. The function analysis step is improved and a new operation "creation and analysis of the function interaction matrix" is added before the trimming step. This improvement highlights the "function streamlines" in the system. This means that the functions are grouped in sets. This idea makes it possible to automate the trimming algorithm and to receive a new concept pattern. 
The rest of the chapter is structured as follows: section 2 is devoted to the state-of-the-art, section 3 describes the method, in section 4 the method is illustrated by an industrial case study, section 5 consists of discussion, and conclusions are presented in section 6 .

\section{State-of-the-art}

The functional part of the presented method is based on TRIZ function analysis. There are several types of function presentation of the system model, such as the Functional Flow Block Diagram (FFBD) (Akiyama 1991), the Functional Analysis System Technique (FAST) (Cooke 2015), the Integrated Computer-Aided Manufacturing Definition for Function Modeling (IDEF0) (Defense Acquisition University 2005). All these methods use the function approach for system model presentation. For example, FFBD is a function-oriented approach based on the sequential relationship of all system functions. FFBD develops the system from the top to the bottom and proposes a hierarchal view of the functions across a series of levels. The aim of each level is to identify a single task on a higher level by means of functional decomposition. In comparison to FFBD, FAST diagram focuses on the product functions rather than a specific design. In contrast to FFBD and FAST, the TRIZ function modeling takes account of the physical interaction between the system elements and the type of this interaction. There are four interaction types: useful, harmful, insufficient, or excessive. FFBD and FAST use the static approach in the function analysis, meaning that the number of elements and functions, and the relation between the elements are time-independent and do not change in time, whereas many TRIZ practitioners point out the need to identify problems clearly at each system level, and to solve them separately. This goal is achieved by integrating well-known models and instruments for system description and function representation. $\mathrm{O}$. and N. Feygenson also suggest the Advanced Function Approach in the Modern TRIZ (Feygenson and Feygenson 2016), where they add some steps, such as: "Indicate the place the function is performed" and "Indicate the time the function is performed". Also this approach has been used by Litvin et al. (2011) in their research in the application history and the function analysis evolution. The research indicates that the next logical step for enhancing the Function Approach is the introduction of two parameters: "time of performing a function" and "place of performing a function". The presented method combines previous works in the domain and proposes taking the physical relation and time-dependence of the system model into account.

The second part of the method consists of trimming. This is a formal process to improve the system model by means of system complexity reduction. There are different approaches in this case. For example, Miao Li's method (Li et al. 2015) is used in the patent around design. It takes account of the importance of each element. The Gen3 method (Ikovenko et al. 2005) is used for design improvement and development. This method uses the formal functional approach to rank the importance of each function in the system. The approach presented in this chapter combines and supplements these methods, and collects previous works in this domain.

\section{Method description}

This chapter contains the method description by using a simple example. The method uses function modeling for system model improvement by means of system complexity reduction. The suggested method consists of two main parts: function analysis and trimming.

\subsection{Function presentation and other definitions}

In TRIZ function modeling, the functions are presented in the following manner: the tool (the function carrier), the function, and the object. The function must create real action from the tool to the object, e.g. "a helmet deflects a bullet" is a legitimate function, but "a helmet 
protects a head" is not a legitimate function. On the other hand, the function does not be declarative, e.g. "a pill improves the health".

The function rank is a formal factor which defines the importance of the function in the function model. In this case, the rank is a positive integer number. As such, the argument of the rank function is inversely proportional to the importance of the function. For example, a function with rank three is more important than a function with rank five.

The ranking factor is a formal index which defines the function rank. It is a rational number and maybe positive or negative. The latter is also inversely proportional to the importance of the function. Thus, a small value defines the most important function.

The target element is called the main element in the system. This means that this element defines the purpose of the system in the initial function model.

The target function is a function which interacts with the target element. On the other hand, this function defines the main function of the initial system.

\subsection{Function analysis}

The function analysis part consists of three main steps: component analysis, interaction analysis and function modeling. Component analysis concerns decomposition of the system model to the main parts, e.g. to big assemblies. This step may be done by means of CAD model analysis (Efimov-Soini and Chechurin 2017). In the interaction analysis step, the interaction between the elements is defined. In the final step, a function diagram (function model) is created and the rank (importance) of the functions in the system is defined.

\subsubsection{Component analysis}

This step concerns system model decomposition. Complex systems are usually divided into big assemblies and simple systems to parts. This analysis includes not only system model parts, but also external parts. For example, a car consists of an engine, a frame, a body etc., but in some cases a road may be included in the system model. The main goal of this step is to create a detailed system decomposition model in the area close to the target element.

\subsubsection{Interaction analysis}

A special interaction matrix is used in the interaction analysis step. In this matrix, the interaction between the elements of the system model is denoted with a plus sign (+) and the lack of interaction with a minus sign (-). As an example, the interaction matrix of the system a cup with a cap - coffee, is presented in Table 8.1. This system model is used below to illustrate the method description. The case study is illustrated with a real industrial case.

Table 8.1 - An interaction matrix

\begin{tabular}{|l|l|l|l|l|}
\hline & cup & table & coffee & cap \\
\hline cup & & + & + & + \\
\hline table & + & & - & - \\
\hline coffee & + & - & & + \\
\hline cap & + & - & + & \\
\hline
\end{tabular}

\subsubsection{Function modeling}

There are six substeps in this step: definition of the interaction as a function, initial ranking, initial function model creation, model selecting, final ranking, and function interaction analysis. 
Firstly, each interaction is defined as a function in the substep called "interaction definition as a function". If interaction between the elements is not available, the function is not defined. The functions in the system model are presented in Table 8.2.

Table 8.2 - Functions in the system model

\begin{tabular}{|l|l|l|l|}
\hline Element 1 & Function & Element 2 & \\
\hline cup & holds & coffee & target function \\
\hline table & holds & cup & \\
\hline cap & holds & coffee & \\
\hline cup & holds & cap & \\
\hline
\end{tabular}

The target function and the target element are also defined in this step. In this case, the target function is "cup holds coffee" and the target element is "coffee".

Next, the initial function rank is defined. The rank (importance) of each function is defined on the interval $(1 \ldots+\infty)$. When the initial rank of the target function is 0 , it is the highest in the system. The initial ranking of the suggested system is presented in Table 8.3.

Table 8.3 - Initial ranking

\begin{tabular}{|l|l|l|l|}
\hline Element 1 & Function & Element 2 & Rank \\
\hline cup & holds & coffee & 0 \\
\hline table & holds & cup & 1 \\
\hline cap & holds & coffee & 1 \\
\hline cup & holds & cap & 1 \\
\hline
\end{tabular}

After this, the function model is created by means of the previous step results. At this point, it is possible to create the function model simultaneously with the initial rank definition. In the function diagram, the elements are marked as rectangles. It is also recommended to place the target element in the right side. In addition, for elements which are impossible to modify or are not included in the model, create action (e.g. gravitation) is noted with a hexagon.

In the suggested method, two model types are presented: static and dynamic. In the static model the function rank (importance), the interaction number in the system, and the number of the elements in the system are permanent. On the other hand, one or all parameters can be changed in a dynamic model. The dynamic model is presented as a set of system snapshots. Each snapshot is a static state of the system, which means that the function rank, the number of interactions and the number of elements are permanent in each state. For each static state, the time (duration) of each snapshot is defined by means of the following formula:

$t_{n i}=\frac{t_{i}}{t_{w}}$

where $t_{n i}$ is the normalized time of the state $\mathrm{i}, t_{i}$ - the time of the state (in minutes, seconds, years etc.) and $t_{w}$ is the total observation time (in minutes, seconds, years etc.).

The presented approach is used to calculate the final function rank. In this case, the special formal index (the ranking factor) is used. The latter is inversely proportional to the importance of the function. Thus, a small value of ranking factor defines the most important function. Using normalized time, the final ranking factor is defined as 


$$
F F R=\sum F R_{i} \times t_{n i}
$$

where $F F R$ is the final ranking factor, $F R_{i}$ is the ranking factor in state $\mathrm{i}$, and $t_{n i}$ is the normalized time of the state $i$. For a static system, $t_{n i}=1$.

For the presented system, both the dynamic and the static approach may be used. For the dynamic approach, two states are considered, the cup is on the table $\left(\operatorname{tn}_{1}=0.9\right)$ and the cup is lying on its side $\left(\operatorname{tn}_{2}=0.1\right)$.

In the final ranking substep, the special index, called the ranking factor, is added to define the function rank. The following formula is used to calculate this:

$F R_{i}=R-N_{l}-N_{d}$

where $F R_{i}$ is the ranking factor in this state, $R$ is the initial rank, $N_{l}$ is the number of function carrier links, $N_{d}$ is the number of duplicated functions. The final ranking is presented in Table 8.4 .

Table 8.4 - Final ranking

\begin{tabular}{|l|l|l|l|l|l|l|l|}
\hline Element1 & Function & Element2 & R & Nl & Nd & FR & Final rank \\
\hline cup & holds & coffee & 0 & 3 & 0 & -3 & 1 \\
\hline table & holds & cup & 1 & 1 & 0 & 0 & 3 \\
\hline cap & holds & coffee & 1 & 2 & 0 & -1 & $2 \mathrm{~B}$ \\
\hline cup & holds & cap & 2 & 3 & 0 & -1 & $2 \mathrm{~A}$ \\
\hline
\end{tabular}

Additional subindexes are used in the suggested case, such as $2 \mathrm{~A}$ and $2 \mathrm{~B}$. The letter is used to distinguish functions with an equal ranking factor. The function with subindex $A$ is geometrically closer than the function with subindex B. This means that the element "cup" is closer to the element "coffee" than the element "cap" to the element "coffee".

The situation is different in the dynamic model, and the situation is different in the dynamic approach as well. There are two system states: the cup is on the table $\left(\operatorname{tn}_{1}=0.9\right)$ and the cup is lying on its side $\left(\operatorname{tn}_{2}=0.1\right)$. The latter state function model is equal to the model in the static approach.

The function "cap holds cup" is not available in this state, because these elements do not interact here. The ranking table for this case is presented below.

Table 8.5 - Ranking in the dynamic approach

\begin{tabular}{|l|l|l|l|l|l|l|l|l|l|l|}
\hline Element1 & Function & Element2 & R1 & R2 & NI1 & Nl2 & Nd & FR1 & FR2 & $\begin{array}{l}\text { Final } \\
\text { rank }\end{array}$ \\
\hline cup & holds & coffee & 0 & 0 & 3 & 3 & 0 & $-3^{*} 0.9$ & $-3 * 0.1$ & 1 \\
\hline table & holds & cup & 1 & 1 & 1 & 1 & 0 & $0 * 0.9$ & $0 * 0.1$ & 4 \\
\hline cap & holds & coffee & 1 & NA & 2 & 2 & 0 & $-1^{*} 0.9$ & $-1^{*} 0.1$ & 3 \\
\hline cup & holds & cap & 2 & 2 & 3 & 3 & 0 & $-1^{*} 0.9$ & $-1 * 0.1$ & 2 \\
\hline
\end{tabular}

Finally, sets of functions are defined after ranking. This approach makes it possible to improve the trimming process and to receive some new design patterns. In this case, the functions are divided into three types: independent (-), dependent $(+)$ and equal (=). Independent functions do not interact - e.g. in the fun system, functions installed in the wall "wall holds fun" and "fun moves air" are independent. In the dependent type, functions create 
the result "together", e.g. "bolt holds nut" and "nut holds plate" are dependent functions. The equal functions create a similar result in the system, e.g. functions "welding holds plate" and "bolt holds plate" are equal in many cases. In this case, independent functions trim in a separate manner, and dependent and similar functions in sets. The function interaction matrix for the presented model is shown in Table 8.6.

Table 8.6 - The function interaction matrix

\begin{tabular}{|l|l|l|l|l|}
\hline & cup holds coffee & table holds cup & cap holds coffee & cup holds cap \\
\hline cup holds coffee & & - & $=$ & - \\
\hline table holds cup & - & & - & - \\
\hline cap holds coffee & $=$ & - & & + \\
\hline cup holds cap & - & - & + & \\
\hline
\end{tabular}

There are two sets of functions, the dependent set "cup holds cap" and "cap holds coffee" and the set of equal functions "cup holds coffee" and "cap holds coffee".

\subsection{Trimming}

Trimming is a formal process to improve the system model by means of system complexity reduction. There are three formal rules. The function may be trimmed if
a) An object of the Function does not exist
b) An object of the Function performs the function itself
c) Another Engineering System Component performs the useful function of the Function Carrier.

The trimming procedure starts with the function with a lower rank. If sets of function are defined in the system model, the trimming process starts with the last one. Three formal rules are used to trim the sets in the trimming process. This is a radical method, but it makes it possible to gain a new qualitative design pattern.

In the presented case the trimming process has the following steps:

- 'Cup holds cap' and 'cap holds coffee' (set "cup holds coffee") may be trimmed in case of the transfer function. For example, the function "holds" is transferred to the table and the new system is a table with a thermos. Another example: the function transfers to coffee itself to create a solid shell of coffee. In this case, the coffee holds itself. This trimming process is similar to the previous one.

- 'Table holds cup'. This function has a lower rank in the system (in the static and dynamic approaches) and maybe trimmed by means of rule B. It is possible to place the cap on the floor or hold it in the hand.

- 'Cap holds coffee' may be trimmed if the function is transferred to the cap (similar to a baby cup). In this case, the element "cap" is trimmed, and as well the function "Cup holds cap" is trimmed as well by means of rule A.

\section{Case study}

The industrial case is presented in this chapter. The case concerns a special tool for flow meter assembling. This procedure is used by the firm Termotronic (Saint-Petersburg, Russia) in the manufacturing process. The presented mechanism is very complex, and therefore this chapter focuses only on the flow meter holding system. The step-by-step algorithm is presented below.

The holding system is presented in Figure 8.1. The system is inspired with a linear actuator and it is used to hold the flow meter on a vertical axis. Considering that the various flow 
meter models have different tube diameters, the system must be adaptive. In this case, a system based in pinions is used. The user rotates a handle, this handle rotates the first pinion, then the pinion rotates the driven pinion, and the last one moves the thread. In this process, the thread moves the holder on the horizontal axis. The frame in this system holds the holder.

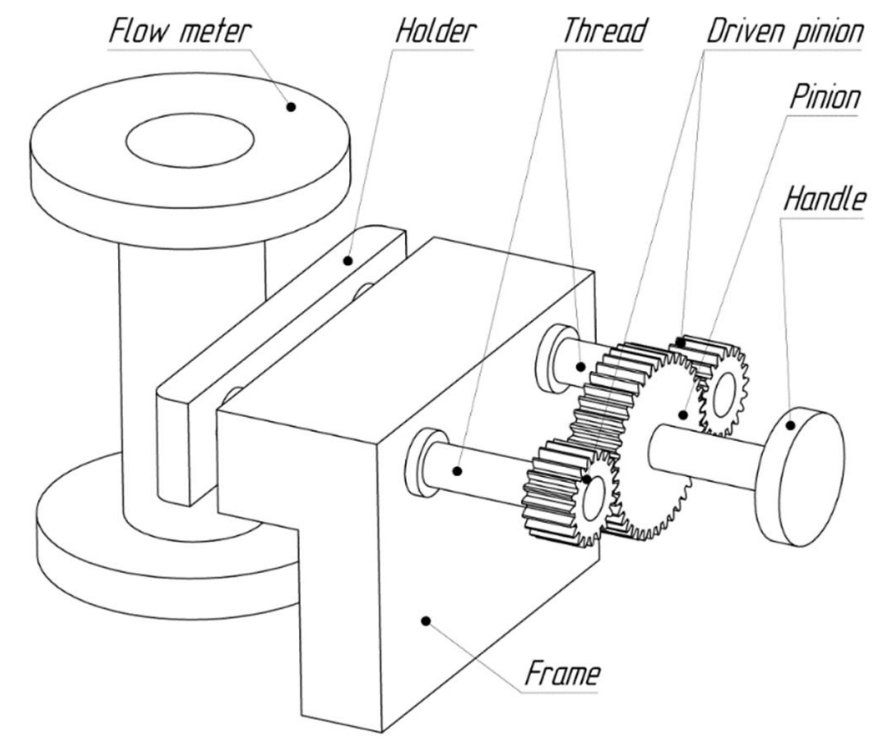

Figure 8.1 - Initial flow meter holding system

\subsection{Function analysis of the holding system}

The first step in the suggested approach is function analysis. This approach is based on previous developments (Efimov-Soini and Chechurin 2016; Efimov-Soini et al. 2016; Efimov-Soini and Uzhegov 2017) and the Gen3 function analysis approach (Ikovenko et al. 2005).

There are three parts on this step: component analysis, interaction analysis and function analysis. The first and second steps are accomplished by means of a special software (EfimovSoini and Chechurin 2017) which uses a CAD model of the flow meter holding system. The function model of the holding system is presented in Figure 8.2. 


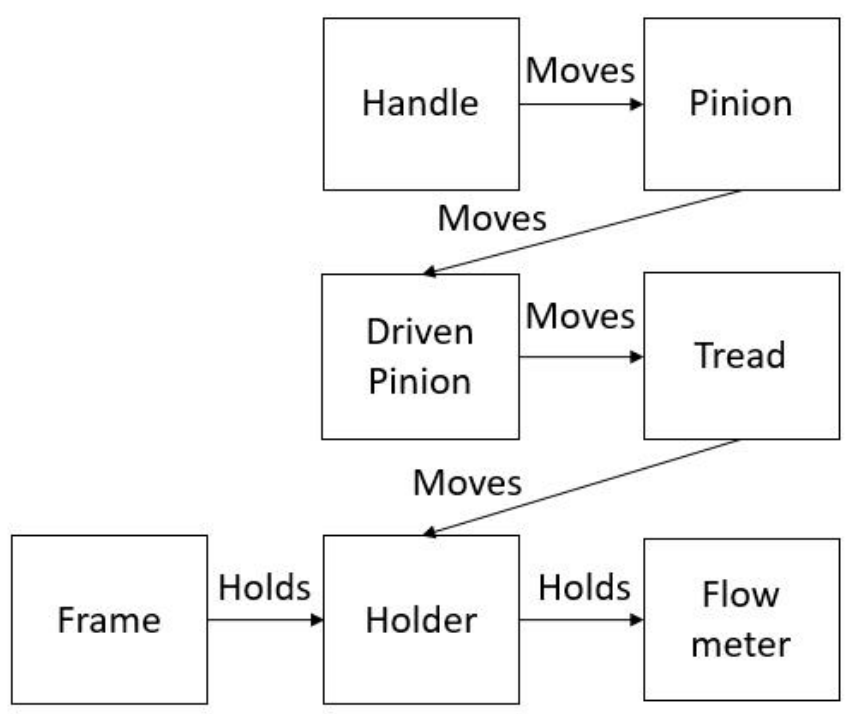

Figure 8.2 - Function model of the holding system

\subsection{System function interaction matrix}

The interaction between the functions and the interaction type are defined in this step. There are three types of the interaction in the suggested method: dependence (+), independence (-) and similarity $(=)$. In Table 8.7, independent and dependent functions are distinguished. By means of this table, it is possible to divide the functions into two parts, the function "Frame holds Holder" and a set of the five remaining functions, named t "Handle moves Holder".

Table 8.7 - Holding system function interaction table

\begin{tabular}{|l|l|l|l|l|l|l|}
\hline & $\begin{array}{l}\text { Handle } \\
\text { moves } \\
\text { Pinion }\end{array}$ & $\begin{array}{l}\text { Pinion moves Driven } \\
\text { driven pinion }\end{array}$ & $\begin{array}{l}\text { Tread moves } \\
\text { pinion } \\
\text { moves Tread }\end{array}$ & Holder & \multicolumn{2}{l}{$\begin{array}{l}\text { Frame } \\
\text { hold Flowholds } \\
\text { meter }\end{array}$} \\
\hline $\begin{array}{l}\text { Handle moves } \\
\text { Pinion }\end{array}$ & + & + & + & + & - \\
\hline $\begin{array}{l}\text { Pinion moves } \\
\text { Driven pinion }\end{array}$ & + & & + & + & + & - \\
\hline $\begin{array}{l}\text { Driven pinion } \\
\text { moves Tread }\end{array}$ & + & + & & + & + & - \\
\hline $\begin{array}{l}\text { Tread moves } \\
\text { Holder }\end{array}$ & + & + & - & & + & - \\
\hline $\begin{array}{l}\text { Holder holds } \\
\text { Flow meter }\end{array}$ & + & + & + & + & & - \\
\hline $\begin{array}{l}\text { Frame holds } \\
\text { Holder }\end{array}$ & - & - & - & - & - & \\
\hline
\end{tabular}

\subsection{Trimming of the system}

The system simplification is completed by means of a trimming algorithm in this step. There the set of functions "Handle moves Holder" and the function carriers of this set are transformed to the system "Handle-Spring-Holder". This means that a spring is added into the system and the functions set "Handle moves Holder" is trimmed by means of Rule C. This system is self-adaptive, which means that the spring holds the flow meter with a different pipe 
diameter without any action. The function model of the improved system is presented in Figure 8.3. The improved system is presented in Figure 8.4.

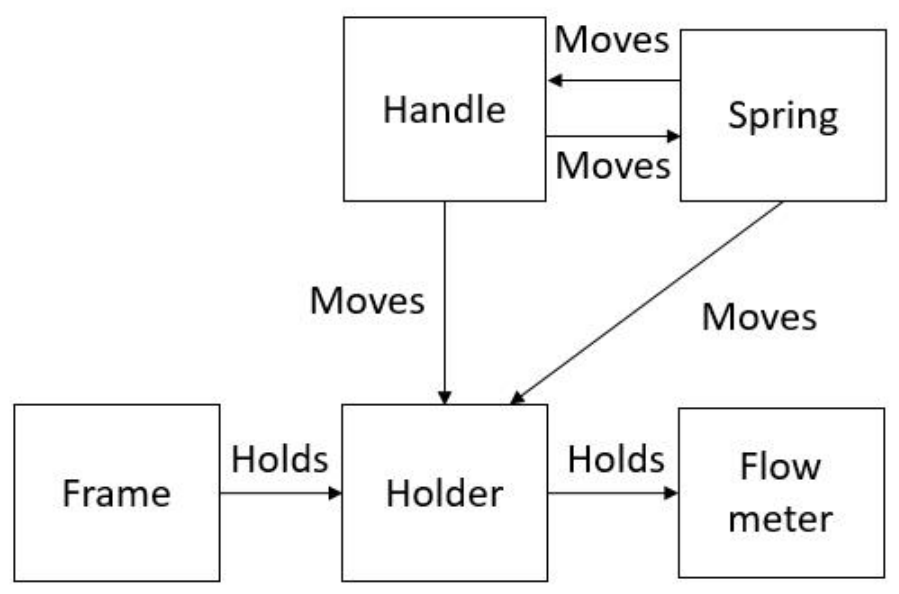

Figure 8.3 - Function model of the improved holding system

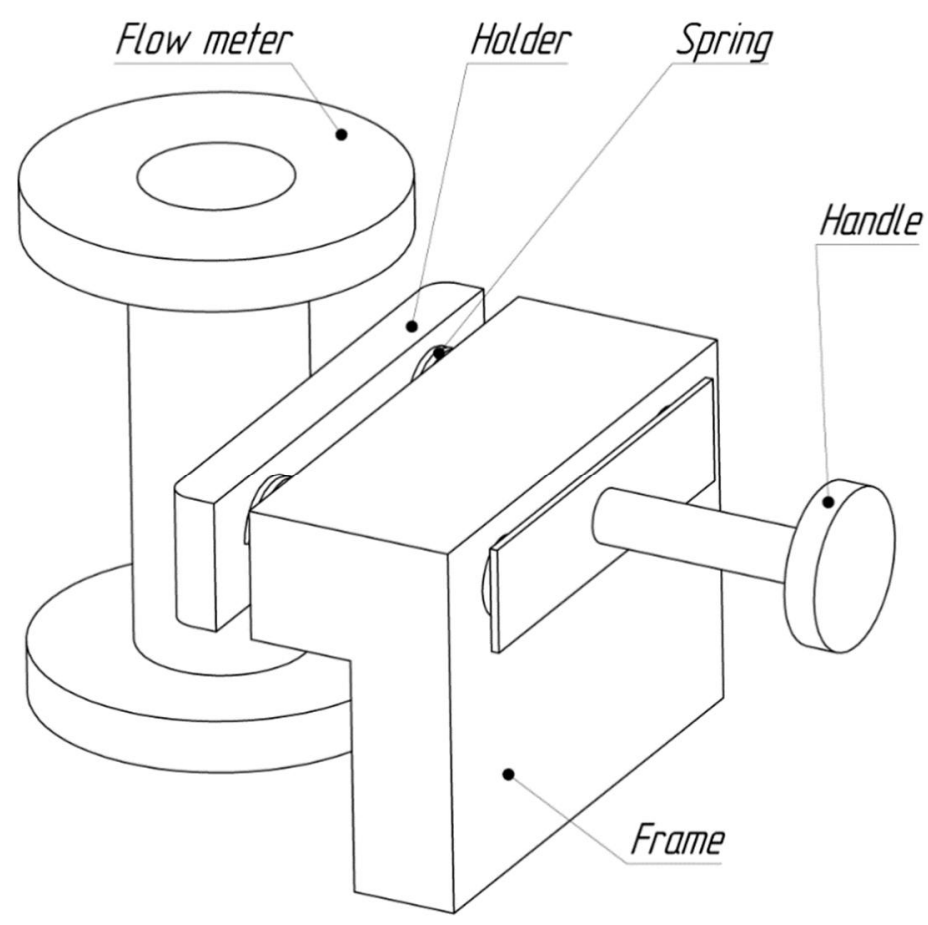

Figure 8.4 - Improved holding system

\section{Discussion}

A special survey was performed to verify the presented algorithm. In this survey, 10 engineers from the firm Termotronic and Institut Telecomunicatsiy improved the existing systems by using the suggested method. They worked manually without any special tool for system decomposition and function ranking to understand the weakness of this approach better. All specialists commended the new design pattern received by means of this method. 8 of the 10 engineers mentioned the difficulty of calculation in complex assemblies and 7 of the 10 mentioned the ambiguity of the functional definition. 


\section{Conclusions}

The presented algorithm makes it possible to improve a system model by means of system function presentation. It takes account of the evolution of the situation (and therefore its function model) and shows how the approach yields trimming ideas that are different from what can be derived from the standard static function ranking procedure. In addition, it is believed that the introduction of the time domain makes function analysis more accurate and realistic. The function analysis is formularized and requires a number of calculations, but makes it possible to create the improvement in a systematic manner.

\section{Acknowledgments}

The authors would like to acknowledge the EU Erasmus plus program and its project Open Innovation Platform for University-Enterprise Collaboration: new product, business and human capital development (Acronym: OIPEC, Grant Agreement No.: 2015-3083/001-001) for the support.

\section{References}

Akiyama, Kaneo. Function Analysis: Systematic Improvement of Quality and Performance. Cambridge MA: Productivity Press Inc., 1991.

Altshuller, Genrich. Creativity as an Exact Science. New York: Gordon and Breach, 1984.

Altshuller, Genrich, and Rafael Shapiro. "Psychology of Inventive Creativity." Issues Psychological 6 (1956): 37-49.

Chechurin, Leonid. "TRIZ in Science. Reviewing Indexed Publications." Procedia CIRP 39 (2016): 156-165. https://doi.org/10.1016/j.procir.2016.01.182.

Cooke, John. "TRIZ-based Modelling and Value Analysis of Products as Processes", Trizfuture 2015, (2015): 1-11.

Defense Acquisition University. System Engineering Fundamentals. Virginia USA: Defense Acquisition University Press, 2005. https://ocw.mit.edu/courses/aeronautics-andastronautics/16-885j-aircraft-systems-engineering-fall2005/readings/sefguide_01_01.pdf.

Efimov-Soini, Nikolai, Leonid Chechurin, Ivan Renev, and Kalle Elfvengren."Method of Time-dependent TRIZ Function Ranking", Procedia CIRP: TRIZ Future 2016.

Efimov-Soini, Nikolai and Leonid Chechurin. "The Method of CAD Software and TRIZ Collaboration." In Creativity in Intelligent Technologies and Data Science, edited by A. Kravets, M. Shcherbakov, M. Kultsova, P. Groumpos, Communications in Computer and Information Science, vol. 754, Springer, Cham (2017): 517-527.

Efimov-Soini, Nikolai and Leonid Chechurin. 2016. "Method of Ranking in the Function Model", Procedia CIRP 39, (2016): 22-26.

Efimov-Soini, Nikolai and Nikita Uzhegov. "The TRIZ-based Tool for the Electrical Machine Development." Progress in Electromagnetics Research Symposium, St Petersburg, Russia, 22-25 May 2017.

Feygenson, Oleg. and Naum Feygenson. "Advanced Function Approach in Modern TRIZ." In 
Research and Practice on the Theory of Inventive Problem Solving (TRIZ). Cham: Springer International Publishing, (2016): 207-221.

Gadd, Karen. TRIZ for Engineers: Enabling Inventive Problem Solving. United Kingdom: Wiley, 2011.

Di Gironimo, Guiseppe, D. Carfora, G. Esposito, C. Labate, R. Mozillo, F. Renno, A. Lanzotti, and M. Suiko. "Improving Concept Design of Divertor Support System for FAST Tokamak Using TRIZ Theory and AHP Approach." Fusion Engineering and Design 88, no. 11 (2013): 3014-3020. https://doi.org/10.1016/j.fusengdes.2013.07.005.

Ikovenko, Sergei, Simon Litvin, and Alex Lyubomirskiy. Basic Training Course, Boston: GEN3 Partners, 2005.

Li, Miao, Xinguo Ming, Lina He, Maokuan Zheng, and Zhitao Xu. "A TRIZ-based Trimming Method for Patent Design Around." CAD Computer Aided Design 62 (May 2015): 20-30. https://doi.org/10.1016/j.cad.2014.10.005.

Litvin, Simon, Naum Feygenson, and Oleg Feygenson. "Advanced Function Approach." Procedia Engineering 9 (2011): 92-102. https://doi.org/10.1016/j.proeng.2011.03.103.

Luo, Yihong, Yunfei Shao, and Ting Chen. "Study of New Wall Materials Design Based on TRIZ Integrated Innovation Method." Management Science and Engineering 6, no. 4 (2012): 15-29. http://dx.doi.org/10.3968/j.mse.1913035X20120604.635.

Sheu, Daniel, and Chun Hou. "TRIZ-based Trimming for Process-machine Improvements: Slit-valve Innovative Redesign." Computers \& Industrial Engineering 66, no. 3 (2013): 555-556. https://doi.org/10.1016/j.cie.2013.02.006.

Sickafus, Ed. Unified Structured Inventive Thinking: How to Invent. USA, MI: Ntelleck, 1997.

Suh, Nam. The Principles of Design. New York: Oxford University Press, 1990.

Ullman, David. The Mechanical Design Process. $4^{\text {th }}$ ed. New York: McGraw-Hill, 2010. 